(Aus dem Pathologischen Institut Marburg. Direktor: Prof. Löhİein)

\title{
Einige bemerkenswerte Beobachtungen zur Pathologie der Grippe ${ }^{1}$ ).
}

Von

\section{Dr. Martha Schmidtmann. \\ Mit 1 Abbildung.}

Von verschiedener Seite liegen bereits ausführliche Beschreibungen der charakteristischen und zugleich mannigfaltigen anatomischen $\mathrm{Be}$ funde bei der diesjährigen Grippeepidemie vor. Da auch wir die gleichen Beobachtungen an unserem Material (150 Fälle) machen konnten, möchte ich diese bekannten Veränderungen nur kurz streifen und mich dann vor allem der Schilderung einiger Besonderheiten zuwenden.

Den konstantesten Befund bildete die eigenartige, vorwiegend hämorrhagische Entzündung der Luftröhre, meist auf deren unterstes Drittel beschränkt oder wenigstens in diesem Teil am stärksten ausgeprägt, und eine gleichartige Erkrankung der Bronchien. In einer Reihe von Fällen kam es außerdem zur Pseudomembranbildung in wechselndem Umfang: bald sind es nur fetzige oder schuppenartige fibrinöse Beläge, die sich leicht mit der Pinzette abziehen lassen, dann wieder liegen dicke fibrinös-eitrige Massen der Innenfläche an, die häufig ähnlich dem Bild der absteigenden Diphtherie das ganze Lumen der Bronchien, besonders der kleinsten, ausfüllen. In einem. Fall fand sich ein derartiger Exsudatausguß der ganzen Luftröhre (S. 232/18.)

AuBerordentlich mannigfaltig sind die Befunde an den Lungen, unter denen wir mehrere typische Bilder unterscheiden können. 1. Gruppe: Während der Juliepidemie sahen wir vorwiegend große, schwere, stark ödematöse Lungen mit umfangreicher, meist die Unterlappen und die hinteren Partien der Oberlappen befallender, konfluierender Bronchiopneumonie, die inflitrierten Partien hatten einen eigenartigen bräunlichen Farbenton. Beim Wiederkehren der Eipidemie traten diese Fälle stark zurück gegenüber einer zweiten Form, bei der vor allem die kleinen Bronchien und Bronchiolen erkrankt waren. Klinisch zeichneten sich diese Fälle durch hochgradigen Stridor aus, der dem bei der Diphtherie so ähnlich war, daß die Tracheotomie wiederholt erwogen wurde.

Den typischsten anatomischen Befund für diese Erkrankungsform bot S. 275/18: Die Lungen sind stark gebläht, auf der Schnittfläche

1) Die Arbeit wurde im. Dezember 1918 abgeschlossen. 
springen die Bronchiolen deutlich vor, sie sind von einem schwarzroten Hof umgeben. Auf Druck entleert sich aus dem Bronchioluslumen ein Tropfen eitrigen Sekrets. Mikroskopisch sind alle kleinen Bronchien mit eiterigem Sekret erfüllt, das reichlich Kokkenhaufen enthält. In der Umgebung der Bronchiolen sind Blutaustritte zu sehen. In den Lungenalveolen findet sich außer Ödem ein eigenartiges, großzelliges Exsudat. - Die anderen Fälle dieser Gruppe zeigten ähnliches Verhalten, verschiedentlich fand sich ein ausgesprochenes interstitielles Emphysem.

Sehr nahe steht dieser Art der Erkrankung die peribronchitische Form; hier haben sich kleine, meist eiterige Entzündungsherde um die Bronchien ausgebildet, oft sind die Bronchien selbst zylindrisch oder sackförmig erweitert, es kommt zur Bildung von Bronchiektasien oder bronchiektatischen Abszessen. Dies führt uns zu den eiterigen Formen der Lungenerkrankungen der Grippe, wo wir die abszedierenden Bronchiopneumonien von den interstitiell phlegmonösen Prozessen trennen müssen.

Schließlich sondern sich von dem bisher Beschriebenen scharf jene eigenartigen, keilförmigen, an der Oberfläche gelegenen Nekrosen und Abszesse ab, wie sie zuerst von Oberndorfer beschrieben wurden. In ihnen finden sich meist die wiederholt beschriebenen peri- und endarteriitischen Veränderungen, die wir wie Borst als sekundär, nicht als primär ansehen möchten.

Meist waren beide Lungen erkrankt, wenn auch nicht in gleicher Ausdehnung und Intensität. Die erkrankten Lungen zeigten fast stets fibrinöse Beläge an ihrer Oberfläche, in vielén Fällen fanden sich eiterige Pleuritiden, deren Exsudat bis zu mehreren Litern betrug.

Die Schwierigkeit in der Beurteilung der Befunde bei der epidemischen Grippe von pathologisch-anatomischer Seite liegt darin, daß wir über die Art des Erregers noch im unklaren sind, wodurch die Betrachtung vom pathogenetischen Standpunkt aus einstweilen unmöglich ist. Es liegt ja auf der Hand, daß man Veränderungen, die lediglich auf die Wirkung des Influenzaerregers zurückzuführen sind, von solchen trennen möchte, die eine typische Komplikation (Mischinfektion) darstellen. Zu letzteren sind wohl alle die verschiedenen Lungenveränderungen zu rechnen, während man geneigt sein wird, die eigenartige hämorrhagische Tracheitis, zum Teil auch Bronchitis, allein der Wirkung des Influenzaerregers zuzuschreiben. (Die gleiche Annahme macht auch Berblinger bei Besprechung seiner Influenzabefunde.) Gestützt wird diese Vermutung durch die bakterioskopische Untersuchung, denn sowohl in Lungenabstrichen wie im Schnitt konnten in den verschiedenen Fällen sehr verschiedene Bakterien nachgewiesen werden. Die häufigsten waren Streptokokken, die besonders in den nekrotischen Lungenbezirkęn, zum 
Teil in langen Ketten, zahllos zu sehen waren. In anderen Schnitten lagen Kokkenkolonien in den bronchiektatisch veränderten Bronchien. Nicht ganz selten waren kleine, zum Teil intrazellulär gelegene, grampositive Diplokokken, und auch Staphylokokken fanden sich in einigen Fällen. Bei nicht die Lungen betreffenden Komplikationen wie Endokarditiden, pyämischen Abszessen, Otitis media, eiteriger Parotitis fanden sich in diesen metastatischen Herden vorwiegend Streptokokken, zum Teil auch kleine Diplokokken, in einem Fall von Endokarditis fusiforme Bakterien. In allen Pleuraexsudaten ließen sich massenhaft Streptokokken nachweisen. Vielleicht ist noch erwähnenswert, daß wir in einem Fall in den Lungenherden eine Komplikation durch AspergillusMykose fanden. Makroskopisch bot S. 331/18 gegenüber anderen Grippefällen keine Besonderheiten: In der Trachea und in den Bronchien fand sich die typische hämorrhagische Entzündung, die Lungen waren von bronchiopneumonischen Herden durchsetzt, die Bronchien zum Teil bronchiektatisch erweitert, an sie schlossen sich eine Anzahl Abszesse an. Mikroskopisch fanden sich im Lumen erweiterter kleiner Bronchien Aspergilluskolonien, die nicht nur auf die Bronchialwand übergegriffenhatten, sondern auch noch in den Lungenalveolen zu erkennen waren.

Gehe ich nun zur Besprechung einiger Besonderheiten über, so möchte ich zunächst einen bei einem Neugeborenen erhobenen Befund mitteilen, der für die Frage der Übertragung des Virus vielleicht eine gewisse Bedeutung hat.

Aus der Krankengeschichte, die Herr Professor Esch mir liebenswürdigerweise zur Verfügung stellte, entnehme ich folgendes: Im Verlauf einer protrahierten Geburt wurde die Kreißende in der Eröffnungsperiode 3 Tage ante partum von einer Influenza befallen. Zunächst herrschten die katarrhalischen Symptome vor, nach 2 Tagen waren geringe bronchiopneumonische Erscheinungen nachweisbar. Wegen Unregelmäßigkeit der beschleunigten kindlichen Herztöne und wegen eines schlechten Allgemeinzustandes der Mutter wurde die Geburt durch Zangenoperation beendigt. Das Kind war $3700 \mathrm{~g}$ schwer und $51 \mathrm{~cm}$ lang. Sofort nach der Entbindung schnappte es nur bisweilen nach Luft, während der Herzschlag kräftig war. Erst in warmen Bade wimmerte es allmählich auf Hautreize hin. Die Atmung blieb aber beschleunigt und mühsam - Flankenatmung; sie wurde immer wieder oberflächlich und ungenügend, wenn sie nicht durch künstliche Maßnahmen unterstützt wurde. So wurde das meist zyanotische Neugeborene durch warmes Baden abwechselnd mit Hautreizen und künstlicher Atmung (Sauerstoffatmung) $81 / 4$ Stunde am Leben erhalten.

Das Kind wurde dem Pathologischen Institut mit der Diagnose: Intrakranielle Blutung überwiesen. 
Die Sektion (S. 299/18.) ergab einen völlig normalen Befund der Schädelhöhle; es war kein freies Blut vorhanden, die Gehirnhäute wie das Gehirn waren intakt. Auch eine kleine, wohl traumatische, prävertebrale Blutung in der Nierengegend konnte nicht als Todesursache angesprochen werden, wohl aber fand sich eine eigenartige Veränderung beider Lungen. Die Lungen haben vermehrtes Volumen und deutlich vermehrte Konsistenz, auf der Schnittfläche, die sehr blutreich ist, finden sich ausgedehnte, luftleere, gekörnte Bezirke, dazwischen lufthaltige Partien. Beide Pleuren sind bedeckt mit leicht abziehbaren, fibrinösen Belägen, sie sind matt. Die Luftröhre sowie die größeren Bronchien haben blasse, glatte Schleimhaut.

Mikroskopisch finden sich in den.Bronchiolen rote Blutkörperchen und Leukocyten, viele etwas größere Bronchien zeigen eine rein eiterige Entzündung. Gruppen von Alveolen sind leukocytär infiltriert, das dazwischenliegende Lungengewebe ist stellenweise emphysematös, an anderen Stellen atelektaktisch. Beide Lungen sind sehr stark hyperämisch. In Abstrichen der Lungenschnittfläche wie auch der Oberfläche fanden sich sehr zahlreich kleine Diplokokken.

Fasse ich den Befunid kurz zusammen, so fand sich bei einem 8 Stunden alten Neugeborenen als Todesursache eine eiterige Bronchitis, bronchiopneumonische Herde in beiden Lungen sowie eine fibrinöse Pleuritis beiderseits. Diese Veränderungen müssen sich bei der kurzen Lebensdauer bereits intrauterin entwickelt haben, wofür auch die Tatsache spricht, daß das Kind bei guter Herztätigkeit asphyktisch geboren wurde, und die Atmung mit allen künstlichen Hilfsmitteln nur kurze Zeit in Gang gebracht werden konnte. Zeichen einer Aspiration ließen sich auch mikroskopisch nirgends nachweisen.

Intrauterine Übertragung ist uns bei luetischen Infektionen ja geläufig; in diesem Fall war bei Untersuchung aller Organe nirgends ein Anhialtspunkt dafür gegeben, ganz abgesehen davon, daß die Pneumoñia alba im allgemeinen sich bereits makroskopisch von der hier beschriebenen Lungenveränderung unterscheidet. Die große Ähnlichkeit, in vielem sogar Übereinstimmung des Lungenbefundes bei diesem Neugeborenen mit den Lungenveränderungen der Grippe der Erwachsenen weist auf die Möglichkeit hin, daß wir es auch hier mit einer entsprechenden Erkrankung zu tun haben. Diese Annahme wird wesentlich gestützt durch die anamnestische Angabe, daß die Mutter 3 Tage ante partum. an schwerer Grippe erkrankte. Ein strikter Beweis für die Richtigkeit unserer Hypothese läßt sich, so lange wir den Influenzaerreger nicht nachweisen können, nicht erbringen.

Die intrauterine UUbertragung ist, dem anatomischen Material nach zu urteilen, selten. Wir hatten wiederholt Gelegenheit, Frühgeburten zu sezieren, bei denen die Mutter an Grippe erkrankt war, in keinem der 
Fälle ließen sich irgendwelche besondere Lungenveränderungen feststellen. Ebensowenig sind solche Befunde in den diesjährigen Mitteilungen über Grippebefunde enthalten. In der älteren Literatur zeigt der von Levy beschriebene Fall (Arch. f. exp. Path. Bd. 26, S. 155) einer intrauterinen Übertragung einer Pneumonie von der Mutter auf das Kind eine große Ähnlichkeit mit dem unseren. Allerdings zeigte das Neugeborene dort erst nach 7 Stunden deutliche Krankheitssymptome (Dyspnoe und Cyanose), während hier das Kind asphyktisch zur Welt kam. Bakteriologisch konnte Le v y beim Kind sowohl im Blut - und zwar in diesem reichlicher - wie in den Lungenherden Pneumokokken nachweisen, was mit dem Befund bei der Mutter übereinstimmte.

Auffallenderweise ist auch in den ausführlicheren Mitteilungen über die Grippeepidemie bei den anatomischen Befunden nirgends etwas von metaplastischen Veränderungen des Bronchialepithels erwähnt, Veränderungen, die wir gar nicht selten beobachten konnten ${ }^{1}$ ). Meist handelte es sich um erweiterte, mit (vorwiegend eitrigem) Exsudat. erfüllte Bronchien. In einigen derartigen Fällen fand sich als Innenauskleidung des ganzen Bronchiallumens ein mehrschichtiges Epithel, das teils nur als Übergangsepithel zu bezeichnen war, teils aber auch den ausgesprochenen Charakter von Plattenepithel hatte. Häufiger als diese Bilder waren Bronchien zu sehen, an deren Innenfläche das Epithel zum Teil fehlte, und die vorhandenen Epithelreste zapfenförmige Bezirke aus geschichtetem, anscheinend in starker Wucherung begriffenen Epithel darstellten. Verhornung fand sich in keinem Fall. Diese Metaplasien traten stets nur an einzelnen Bronchialästen auf, nie war das ganze System von Bronchien in gleicher Weise in allen seinen Teilen beteiligt. Außerordentlich nahe steht diesen Prozessen ein Befund, der bei einem 31jährigen an Grippepneumonie verstorbenen Mann erhoben werden konnte (S. 385/18.).

Der makroskopische Befund an den Lungen entspricht den gewöhnlichen Grippefällen: Beide Lungen sind schwer, in den vorderen Partien stark gebläht, beide Unterlappen sowie die nach hinten gelegenen Bezirke des Mittel- und des Oberlappens sind an der Oberfläche dunkelblaurot gefärbt, auf der Schnittfläche finden sich in diesem Bereich über die gaṇze Schnittfläche ausgesät, gelbe, leicht erhabene, um die kleinsten Bronchien gruppierte Herdchen, die luftleer sind. Das dazwischenge-

1) Inzwischen sind von Schweizer Autoren (Korrespondenzbl. f. Schweiz. Ärzte 1919, 3 und 15) ähnliche Beobachtungen mitgeteilt worden. Fritz s che erwähnt kurz karzinomähnliche, zapfenartige Wucherungen in die Lungenalveolen hinein. Askanazy beschreibt metaplastische Bildung von Plattenepithel an Stellen, an denen das Epithel zerstört war. Er sieht derartige Bildungen sowohl an den Bronchien wie in den Alveolen. 
legene Lungengewebe ist stark durchfeuchtet, dunkelrot gefärbt. Die Bronchien haben dunkelrote Schleimhaut, enthalten rötliches Sekret.

Aus dem mikroskopischen Bild geht hervor, daß es sich bereits um eine vorgeschrittene Veränderung handelt: Die Alveolen sind weit und mit Exsudat gefüllt, das außer zahłreichen Erythrocyten, Leukocyten und abgestoßenen Alveolarepithelien sehr reichlich Fibroblasten enthält. Die Alveolarwände sind an den meisten Stellen von ihrem Epithel entblößt. Die Bronchien sind meist mit eiterigem Exsudat gefüllt, sie sind erweitert und an vielen Stellen ihre Wand eingeschmolzen. Wo der Epithelïberzug der Bronchialwand erhalten ist, ist derselbe so gut wie überall umgewandelt in ein mehrschichtiges Plattenepithel, das an einzelnen Stellen den ganzen Bronchialumfang einnimmt, an anderen finden sich nur die in dem Vorhérgehenden bereits geschilderten Epithelzapfen. Einige Bronchien haben umschriebene, sich in die Umgebung fingerförmig vorschiebende Erweiterungen, die vollkommen von dem gewucherten Epithel eingenommen werden. Nun fällt bereits bei schwacher Vergrößerung auf, daß die Alveolen in der Umgebung einiger größerer Bronchien einen besonderen zelligen Inhalt haben. Sie sind vollkommen ausgefüllt von dicht aneinander gelagerten, vielgestaltigen Zellen mit bläschenförmigem Kern und hellem Protoplasma. Meist liegen die Zellen in Form solider Zapfen zusammen und füllen das ganze Alveolarlumen aus, manchmal enthalten sie im Zentrum einen mit Leukocyten gefüllten Hohlraum. Es ist zweifellos, daß es sich hier um Plattenepithelwucherungen handelt, und es entsteht die Frage, ob diese Wucherung vom Alveolar- oder Bronchialepithel ausgeht. Da sich in dieser Weise veränderte Alveolen nur in der Umgebung von Bronchien finden, ferner die Zapfen in den Alveolen vollkommen denen entsprechen, die wir vom Bronchialepithel ausgehen sahen, schließlich die beschriebenen Zapfen wohl die Alveole vollkommen ausfüllen, aber keinen innigen Zusammenhang mit der Alveolarwand zeigen, so glaube ich zu der Annahme berechtigt zu sein, daß diese Epithelzapfen vom Bronchus in die vom Epithel entblöBte Alveole eingewandert sind.

Von anatomischen Befunden sind noch die ziemlich häufigen Knochenmarkriesenzellen-Embolien zu erwähnen, die sich vorwiegend bei sehr jugendlichen Individuen finden. Das Knochenmark selbst ist in einigen Fällen ausgesprochen rot gefärbtes Fettmark.

In einem Fall konnte ich die Sektion einer $\mathbf{3}$ Wochen nach überstandener schwerer Grippepneumonie an Erysipel verstorbenen. Patientin vornehmen (S. 434/18). Makroskopisch waren die Lungen wenig emphysematös, mikroskopisch fanden sich einige atelektatische Bezirke neben Emphysem, die Bronchiolen zeigten eine geringe Erweiterung und Reste eiteriger Bronchiolitis, das Epithel ist überall intakt, nirgends etwa an Bronchiolitis obliterans erinnernde Veränderungen.

Virchows Archiv. Bd. 228. 
50 Martha Schmidtmann: Einige bemerkenswerte Untersuchungen usw.

An nicht die Lunge betreffenden Grippekomplikationen konnten wir alle die oben bereits aufgezählten beobachten, aus denen sich nur ein Fall besonders heraushebt (S. 383/18). Bei einem etwa 20 jährigen Mädchen mit sehr ausgedehnter abszedierender Bronchopneumonie beider Lungen entwickelte sich im Anschluß an eine metastatische, eiterige Parotitis eine ausgedehnte Thrombose von cerebralen Venen, die die entsprechende, nämlich linke Vena meningea media, den Sinus longitudinalis, den Sinus cavernosus, beide Sinus petrosi superficialis sowie (von der Sinusthrombose fortgeleitet) eine Anzahl pialer Venen

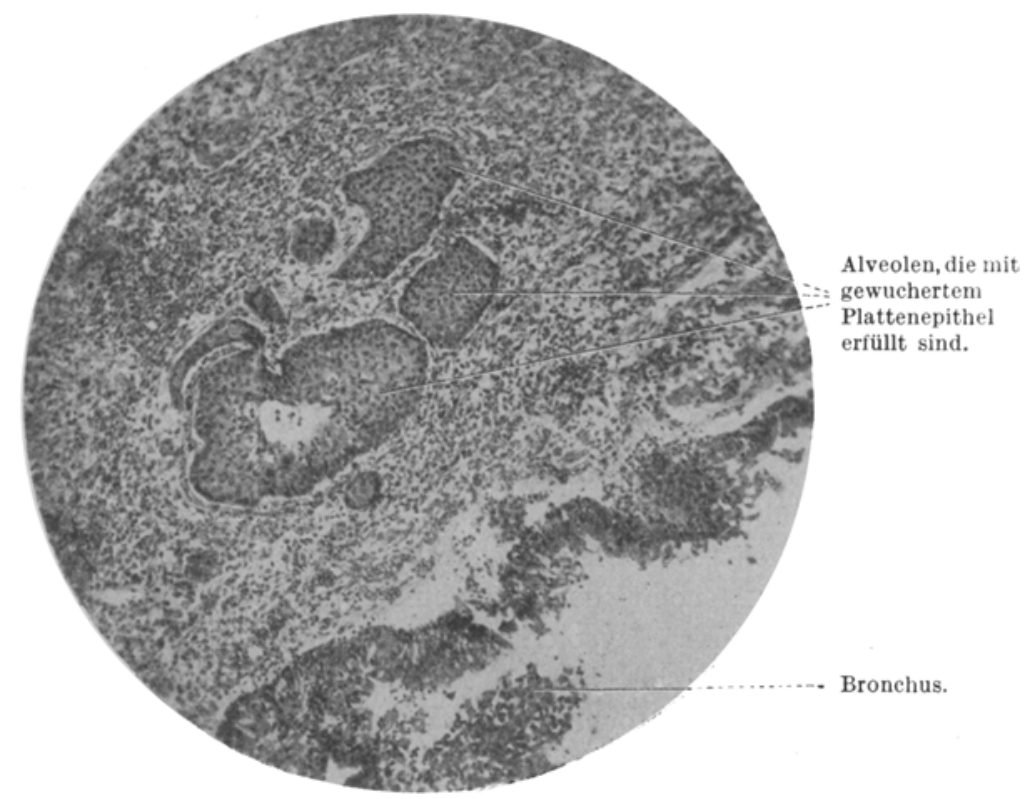

der linken Hemisphäre betraf. Die Thrombosen waren zum Teil eiterig. Am vorderen Pol der linken Hemisphäre fand sich eine umschriebene, eiterige Meningitis mit geringer Menge eiterigen Exsudats. Der Innenfläche der Dura lagen zarte, stark vaskularisierte Membranen auf. Es waren also Thrombosen von einer ganz ungewöhnlichen Ausdehnung vorhanden, was besonders deshalb hervorzuheben ist, als bei der Grippe Thrombosen überhaupt nur selten zur Beobachtung kamen. 